\title{
FCGR2A, FCGR3A polymorphisms and therapeutic efficacy of anti-EGFR monoclonal antibody in metastatic colorectal cancer
}

\author{
Hou-Qun Ying ${ }^{1,2, *}$, Feng Wang ${ }^{1, *}$, Xiao-Lin Chen ${ }^{4, *}$, Bang-Shun $\mathrm{He}^{1}$, Yu-Qin Pan ${ }^{1}$, \\ Jie Chen ${ }^{1,3}$, Xian Liu ${ }^{1}$, Wei-Jun $\mathrm{Cao}^{5}$, Hong-Xin Peng ${ }^{2}$, Kang Lin ${ }^{1}$, Shu-Kui Wang ${ }^{1}$ \\ ${ }^{1}$ Central Laboratory, Nanjing First Hospital, Nanjing Medical University, Nanjing 210006, Jiangsu, China \\ ${ }^{2}$ Medical College, Southeast University, Nanjing 210009, Jiangsu, China \\ ${ }^{3}$ Life Scientific College, Nanjing Normal University, Nanjing 210046, Jiangsu, China \\ ${ }^{4}$ Department of Clinical Laboratory, Pingxiang People's Hospital, Pingxiang, 337055, Jiangxi, China \\ ${ }^{5}$ Department of Digestion, Nanjing First Hospital, Nanjing Medical University, Nanjing 210006, Jiangsu China \\ *These authors have contributed equally to this work
}

Correspondence to:

Shu-Kui Wang, e-mail: shukuiwang@163.com

Keywords: H131R, F158V, FCGR, MCRC, anti-EGFR mAb

Received: April 30, $2015 \quad$ Accepted: August 05, $2015 \quad$ Published: August 19, 2015

\section{ABSTRACT}

Anti-EGFR monoclonal antibodies (mAb) such as cetuximab, panitumumab are one kind of efficacious targeted drugs in treatment of metastatic colorectal cancer ( $\mathrm{MCRC}$ ). However, only a small proportion of patients harbored wild-KRAS genotype can benefit from it. We hypothesized that personal genetic heterogeneity might be the main cause leading to obvious difference in its clinical efficacy. A retrospective study including 82 mCRC patients treated with chemotherapy plus cetuximab and a comprehensive meta-analysis containing 2831 cases within sixteen eligible studies were conducted to investigate the possible association between FCGR2A H131R and FCGR3A V158F and clinical outcome of mCRC patients treated with anti-EGFR mAb based therapy. Results of the retrospective study showed that H131R within FCGR2A or V158F within FCGR3A were not associated with clinical outcome in 82 KRAS wild chemorefractory mCRC patients in co-dominant, dominant, recessive, over-dominant, allele genetic models. However, the comprehensive meta-analysis with the largest of sample size obtained the significant result between FCGR3A V158F and PFS (FV/VV vs. FF: $P_{\mathrm{h}}=0.027, \mathrm{MSR}=0.680,95 \% \mathrm{CI}=0.549-0.842$ in overall population; $\boldsymbol{P}_{\mathrm{h}}=0.12$, MSR $=0.728,95 \% \mathrm{CI}=0.648-0.818$ in KRAS wild population) and OS (VV vs. FF: $P_{\mathrm{h}}<0.001, \mathrm{MSR}=0.733,95 \% \mathrm{CI}=0.578-0.930$ in overall population $)$. These findings indicate that KRAS wild chemorefractory MCRC individual harbored genotype FF of V158Fcan benefit from anti-EGFR mAb adjuvant therapy in terms of PFS and OS, and it may be useful genetic biomarker to predict clinical survival of $\mathbf{m C R C}$ individuals with anti-EGFR mAb based therapy.

\section{INTRODUCTION}

Colorectal cancer (CRC) is one of the most common lethal malignancies in the United States and China $[1,2]$. With limitation of useful early diagnostic biomarker and invasiveness of colonoscopy, most of cases are clinical confirmed as TNM II-IV patients, and mid-term patients can benefit from regular surgical operation plus adjuvant chemoradiotherapy. Meanwhile, the therapeutic options against metastatic CRC (mCRC) have expanded with the introduction of anti-epidermal growth factor receptor monoclonal antibody (anti-EGFR mAb) and treatment outcomes have improved in the past decade [3].

Cetuximab, a chimeric immunoglobulin 1 monoclonal antibody that targets the extracellular domain of the EGFR, has been validated to be effective in providing clinical benefit in wild-KRAS mCRC patients in combination with chemotherapy as first and second 
lines as well as in monotherapy as third line drug [3-7]. However, only approximately $10 \sim 20 \%$ of patients with chemorefractory mCRC derived good clinical benefit from cetuximab therapy [8], revealing that personalized difference in genetic background might affect individual's response and additional mechanisms could lead to CRC progression [9]. Therefore, an understanding of molecular basis of clinical response to cetuximab may be better to identify the subpopulation of patients who are likely to benefit from cetuximab and avoid unnecessary drug toxicity and costs.

The molecular mechanisms underlying response to cetuximab are still substantially unclear; it is that cetuximab acts by means of inhibition of EGFR signal pathway or activation of antibody-dependent cell cytotoxicity (ADCC) [10]. However, only 30 40\% nonresponsive patients harbored $K R A S$ mutation [11, 12], and some KRAS mutated patients also showed to respond to cetuximab [13]. These exacting evidences suggested that ADCC might be involved in cetuximab enhanced antitumor efficacy [13]. ADCC is stimulated through the interaction between Fc fragment of lgG1 monoclonal antibodies linked with EGFR of targeted cancer cell and the surface $\mathrm{Fc}$ gamma receptor $2 \mathrm{a}$ and 3a (FCGR2A and FCGR3A) of IgG carried by immune cells such as natural killer lymphocytes (NK), macrophages and neutrophil, triggering the activation of these immune effective cells and leading to the lysis and death of targeted cancer cell. Genetic variations within FCGR $2 A$ and FCGR $3 A$ may contribute to abnormal secondary spatial structure and function of the products, leading to different binding affinity to cetuximab. H131R and V158F are two common single nucleotide polymorphisms (SNPs) which are located in the third and fifth exon of $F C G R 2 A$ and $F C G R 3 A$, respectively, and two loci appear to be associated with clinical outcome in hematological malignancies and breast cancer with treatment of rituximab and trantuzumab, respectively $[14,15]$. Follicular lymphoma patients harbored allele $\mathrm{V}$ and genotype $\mathrm{VV}$ of $\mathrm{V} 158 \mathrm{~F}$ within FCGR $3 A$ could benefit from the combination therapy including rituximab [16] and genotype $\mathrm{HH}$ of H131R within FCGR2A was significantly associated with a shorter event-free survival in breast cancer patients with sequentially given transtuzumab in UNICANCERPASCO4 trial [17]. Some studies attempted to investigate the role of the two SNPs in treatment efficacy of anti-EGFR mAb in advanced CRC patients, however, these results weren't in consistence with each other [18-20].

In this study, we aimed to investigate the association of FCGR2A $\mathrm{H} 131 \mathrm{~A}$ and FCGR $3 A \mathrm{~V} 158 \mathrm{~F}$ with clinical outcome of 82 wild-KRAS chemorefractory mCRC patients undergoing cetuximab adjuvant therapy. Additionally, a comprehensive meta-analysis including prospective and retrospective studies was carried out to confirm the clinical finding.

\section{RESULT}

Overall, a total of 46 male and 36 female chemorefractory mCRC individuals harbored wide$K R A S$ were included in our study. 52 and 30 were colon and rectal cancer patients, respectively. All of them were TNM-IV stage patients and treated with chemotherapy plus cetuximab. However, only 6 CR, 44 PR, 15 SD and $17 \mathrm{PD}$ were observed in $82 \mathrm{mCRC}$ individuals, respectively. The genotype distributions of H131R within $F C G R 2 A$ and $\mathrm{V} 158 \mathrm{~F}$ within $F C G R 3 A$ were in Hardy-Weinberg equilibrium ( $P=0.52$ for $F C G R 2 A$, and $P=0.09$ for $F C G R 3 A$, respectively) (Table 1 ).

The influence of the two SNPs on the clinical efficacy and survival were described in Table 2 and 3. H131R within $F C G R 2 A$ weren't associated with ORR $(P=0.542$ for HR vs. HH; $P=0.357$ for RR vs. HH; $P=0.454$ for HR/ RR vs. HH; $P=0.598$ for RR vs. HH/HR; $P=0.710$ for HR vs. HH/RR; $P=0.409$ for R vs. H) and DCR $(P=0.644$ for HR vs. HH; $P=0.461$ for RR vs. HH; $P=0.559$ for HR/RR vs. HH; $P=0.527$ for RR vs. HH/HR; $P=0.787$ for HR vs. HH/RR; $P=0.510$ for R vs. H) in co-dominant, dominant, recessive, over-dominant and allele genetic models, respectively. No statistical significant difference in response to cetuximab based therapy $(P=0.425$ for $\mathrm{FV}$ vs. FF; $P=0.835$ for VV vs. FF; $P=0.454$ for $\mathrm{FV} / \mathrm{VV}$ vs. FF; $P=0.967$ for VV vs. FF/FV; $P=0.441$ for $\mathrm{FV}$ vs. $\mathrm{FF} / \mathrm{VV} ; P=0.535$ for V vs. F) or DCR $(P=0.463$ for $\mathrm{FV}$ vs. FF; $P=0.957$ for VV vs. FF; $P=0.559$ for $\mathrm{FV} / \mathrm{VV}$ vs. FF; $P=1.000$ for VV vs. FF/FV; $P=0.446$ for FV vs. $\mathrm{FF} / \mathrm{VV} ; P=0.718$ for V vs. F) based on $F C G R 3 A$ V158F was observed. Also, there was no significant association between FCGR combined genotype and ORR $(P=0.642$ for RR or VV vs. $\mathrm{H}$ and $\mathrm{F})$ and DCR $(P=0.554$ for RR or VV vs. $\mathrm{H}$ and F) in present study.

The median PFS of cases harbored allele $\mathrm{R}$ and $\mathrm{H}$ within H131R of FCGR2A were 6.0 and 6.0 months, and 5.5, 7.0, 6.0, 6.0, 6.0, 6.0 months for genotype HH, HR, RR, HR/ RR, HH/HR, HH/RR of FCGR $2 A$, respectively. However, H131R wasn't associated with PFS in co-dominant $(\mathrm{HR}=1.086,95 \% \mathrm{CI}=0.636-1.856$ for $\mathrm{HR}$ vs. $\mathrm{HH}$; $\mathrm{HR}=0.608,95 \% \mathrm{CI}=0.203-1.816$ for $\mathrm{RR}$ vs. $\mathrm{HH})$, dominant $(\mathrm{HR}=1.02,95 \% \mathrm{CI}=0.608-1.713)$, recessive $(\mathrm{HR}=0.636,95 \% \mathrm{CI}=0.223-1.815)$, over-dominant $(\mathrm{HR}=1.162,95 \% \mathrm{CI}=0.687-1.964)$ and allele $(\mathrm{HR}=0.989$, $95 \% \mathrm{CI}=0.733-1.333)$ models. The median OS of cases carrying H131R genotypes and alleles was 13 months, and there was no significant difference in OS in comparison of $\mathrm{HR}$ vs. $\mathrm{HH}(\mathrm{HR}=1.332,95 \% \mathrm{CI}=0.765-2.318)$, RR vs. $\mathrm{HH}(\mathrm{HR}=1.341,95 \% \mathrm{CI}=0.474-3.797), \mathrm{HR} / \mathrm{RR}$ vs. $\mathrm{HH}(\mathrm{HR}=1.329,95 \% \mathrm{CI}=0.779-2.269)$, RR vs. $\mathrm{HR} /$ $\mathrm{HH}(\mathrm{HR}=1.233,95 \% \mathrm{CI}=0.475-3.203), \mathrm{HR}$ vs. $\mathrm{HH} /$ 
Table 1: Baseline characteristics of $82 \mathrm{mCRC}$ patients treated with adjuvant cetuximab

\begin{tabular}{|c|c|c|}
\hline Characteristic & No. of patients & $\%$ \\
\hline Median and range of age (years) & $61(51-70)$ & \\
\hline \multicolumn{3}{|l|}{ Gender } \\
\hline Male/female & $46 / 36$ & $56.1 \% / 43.9 \%$ \\
\hline \multicolumn{3}{|l|}{ Location } \\
\hline Left/right colon/rectal & $25 / 27 / 30$ & $30.5 \% / 32.9 \% / 36.6 \%$ \\
\hline \multicolumn{3}{|l|}{ Treatment } \\
\hline Cetuximab + CapeOX & 8 & $9.76 \%$ \\
\hline Cetuximab + CapeOX+AVASTIN & 2 & $2.43 \%$ \\
\hline Cetuximab +FOLFIR 1 & 19 & $23.17 \%$ \\
\hline Cetuximab +FOLFIR1+AVASTIN & 1 & $1.22 \%$ \\
\hline Cetuximab +FOLFIRI+ Capecitabine & 1 & $1.22 \%$ \\
\hline Cetuximab+FOLFOX & 42 & $51.23 \%$ \\
\hline Cetuximab+FOLFOX+FOLFIRI & 5 & $6.10 \%$ \\
\hline Cetuximab+FOLFOX+AVASTIN & 1 & $1.22 \%$ \\
\hline Cetuximab+Tegafur & 1 & $1.22 \%$ \\
\hline Cetuximab+Capecitabine & 2 & $2.43 \%$ \\
\hline \multicolumn{3}{|l|}{ Tumor response } \\
\hline $\mathrm{CR} / \mathrm{PR}$ & $6 / 44$ & $7.32 \% / 53.66 \%$ \\
\hline $\mathrm{SD} / \mathrm{PD}$ & $15 / 17$ & $18.29 \% / 20.73 \%$ \\
\hline \multicolumn{3}{|l|}{$F C G R 2 A \mathrm{H} 131 \mathrm{R}$} \\
\hline $\mathrm{HH} / \mathrm{HR} / \mathrm{RR}$ & $33 / 44 / 5$ & $40.24 \% / 53.66 \% / 6.10 \%$ \\
\hline \multicolumn{3}{|l|}{$F C G R 3 A$ F $158 \mathrm{~V}$} \\
\hline $\mathrm{FF} / \mathrm{FV} / \mathrm{VV}$ & $49 / 25 / 8$ & $59.76 \% / 30.49 \% / 9.75 \%$ \\
\hline
\end{tabular}

Abbreviation: mCRC: metastatic colorectal cancer; CR: complete response; PR: partial response; SD: stable disease; PD: progressive disease; CapeOX: capecitabine plus oxaliplatin; FOLFIR1: 5-fluorouracil plus calcium folinate plus irinotecan; FOLFOX: 5-fluorouracil plus calcium folinate plus oxaliplatin.

Table 2: Clinical response of wild-KR $A S \mathrm{mCRC}$ patients treated with adjuvant cetuximab according to FCGR2A and FCGR3A polymorphisms

\begin{tabular}{|c|c|c|c|c|c|c|c|c|}
\hline \multirow{2}{*}{$\begin{array}{l}\text { Genetic } \\
\text { Model }\end{array}$} & \multirow{2}{*}{ Polymorphism } & \multirow{2}{*}{$\begin{array}{c}\text { No. of } \\
\text { patients }\end{array}$} & \multicolumn{3}{|c|}{ Objective response } & \multicolumn{3}{|c|}{ Disease control } \\
\hline & & & $\mathrm{CR}+\mathrm{PR}$ & SD+PD & $P$-value & $\mathrm{CR}+\mathrm{PR}+\mathrm{SD}$ & PD & $P$-value \\
\hline & FCGR2A H131R & & No. & No. & & No. & No. & \\
\hline \multirow[t]{2}{*}{ Co-dominant } & HR vs. HH & $44 / 33$ & $12 / 7$ & $32 / 26$ & 0.542 & $21 / 14$ & $23 / 19$ & 0.644 \\
\hline & RR vs. HH & $5 / 33$ & $2 / 7$ & $3 / 26$ & $0.357^{*}$ & $3 / 14$ & $2 /-19$ & $0.461^{*}$ \\
\hline Dominant & HR/RR vs. HH & $49 / 33$ & $14 / 7$ & $35 / 26$ & 0.454 & $24 / 14$ & $25 / 19$ & 0.559 \\
\hline Recessive & RR vs. HH/HR & $5 / 77$ & $2 / 19$ & $3 / 58$ & $0.598^{*}$ & $3 / 35$ & $2 / 42$ & $0.527^{*}$ \\
\hline $\begin{array}{l}\text { Over- } \\
\text { dominant }\end{array}$ & HR vs. HH/RR & $44 / 38$ & $12 / 9$ & $32 / 29$ & 0.71 & $21 / 17$ & $23 / 21$ & 0.787 \\
\hline Allele & R vs. H & $54 / 110$ & $16 / 26$ & $38 / 84$ & 0.409 & $27 / 49$ & $27 / 61$ & 0.51 \\
\hline
\end{tabular}

(Continued) 


\begin{tabular}{|c|c|c|c|c|c|c|c|c|}
\hline \multirow{2}{*}{$\begin{array}{c}\text { Genetic } \\
\text { Model }\end{array}$} & \multirow{2}{*}{ Polymorphism } & \multirow{2}{*}{$\begin{array}{c}\text { No. of } \\
\text { patients }\end{array}$} & \multicolumn{3}{|c|}{ Objective response } & \multicolumn{3}{|c|}{ Disease control } \\
\hline & & & $\mathrm{CR}+\mathrm{PR}$ & SD+PD & $P$-value & $\mathrm{CR}+\mathrm{PR}+\mathrm{SD}$ & PD & $P$-value \\
\hline & FCGR3A F158V & & & & & & & \\
\hline \multirow[t]{2}{*}{ Co-dominant } & FV vs. FF & $25 / 49$ & $5 / 14$ & $20 / 35$ & 0.425 & $10 / 24$ & $15 / 25$ & 0.463 \\
\hline & VV vs. FF & $8 / 49$ & $2 / 14$ & $6 / 35$ & $0.835^{*}$ & $4 / 24$ & $4 / 25$ & $0.957^{*}$ \\
\hline Dominant & FV/VV vs. FF & $33 / 49$ & $7 / 14$ & $26 / 35$ & 0.454 & $14 / 24$ & $19 / 25$ & 0.559 \\
\hline Recessive & VV vs. FF/FV & $8 / 74$ & $2 / 19$ & $6 / 55$ & $0.967^{*}$ & $4 / 34$ & $4 / 40$ & $1.000^{*}$ \\
\hline $\begin{array}{l}\text { Over- } \\
\text { dominant }\end{array}$ & FV vs. FF/VV & $25 / 57$ & $5 / 16$ & $20 / 41$ & 0.441 & $10 / 28$ & $15 / 29$ & 0.446 \\
\hline \multirow[t]{3}{*}{ Allele } & V vs. F & $41 / 123$ & $9 / 33$ & $32 / 90$ & 0.535 & $18 / 58$ & $23 / 65$ & 0.718 \\
\hline & FCGR combined & & & & & & & \\
\hline & $\begin{array}{c}\mathrm{H} \text { and } \mathrm{F} \text { vs RR } \\
\text { or VV }\end{array}$ & $69 / 13$ & $17 / 4$ & $52 / 9$ & 0.642 & $31 / 7$ & $38 / 6$ & 0.554 \\
\hline
\end{tabular}

Abbreviation: mCRC: metastatic colorectal cancer; CR: complete response; PR: partial response; SD: stable disease; PD: progressive disease. $P$-value: result of chi-square test;

${ }^{*} P$-value: result of fisher's exact test.

Table 3: The polymorphisms of $F C G R 2 A$ and $F C G R 3 A$ and clinical survival of 82 wild-KRAS mCRC patients treated with adjuvant cetuximab

\begin{tabular}{|c|c|c|c|c|c|c|c|c|c|c|c|}
\hline \multirow{3}{*}{ Model } & \multirow{3}{*}{ Locus } & \multicolumn{5}{|c|}{ Progression-free survival } & \multicolumn{5}{|c|}{ Overall survival } \\
\hline & & \multicolumn{2}{|c|}{ Months } & \multirow{2}{*}{$\begin{array}{c}P- \\
\text { value }^{*}\end{array}$} & \multicolumn{2}{|r|}{ Cox } & \multicolumn{2}{|c|}{ Months } & \multirow{2}{*}{$\begin{array}{c}P- \\
\text { value }^{*}\end{array}$} & \multicolumn{2}{|r|}{ Cox } \\
\hline & & Median & $95 \% \mathrm{CI}$ & & $\mathbf{H R}^{\#}$ & $95 \% \mathrm{CI}$ & Median & $95 \% \mathrm{CI}$ & & $\mathbf{H R}^{\#}$ & $95 \% \mathrm{CI}$ \\
\hline \multicolumn{12}{|c|}{ FCGR2A H131R } \\
\hline \multirow[t]{2}{*}{ Co-dominant } & $\begin{array}{c}\text { HR } \\
\text { vs. HH }\end{array}$ & $7 / 5.5$ & $\begin{array}{c}5.22-8.78 / \\
1.27-9.73\end{array}$ & 0.937 & 1.086 & $0.636-1.856$ & $13 / 13$ & $\begin{array}{c}10.54-15.46 / \\
8.43-17.57\end{array}$ & 0.642 & 1.332 & $0.765-2.318$ \\
\hline & $\begin{array}{c}\text { RR } \\
\text { vs. HH }\end{array}$ & $6 / 5.5$ & $\begin{array}{l}3.52-8.48 / \\
1.27-9.73\end{array}$ & 0.675 & 0.608 & $0.203-1.816$ & $13 / 13$ & $\begin{array}{c}11.76-14.24 / \\
8.43-17.57\end{array}$ & 0.247 & 1.341 & $0.474-3.797$ \\
\hline Dominant & $\begin{array}{l}\text { HR/RR } \\
\text { vs. HH }\end{array}$ & $6 / 5.5$ & $\begin{array}{c}5.19-6.81 / \\
1.27-9.73\end{array}$ & 0.996 & 1.02 & $0.608-1.713$ & $13 / 13$ & $\begin{array}{c}11.80-14.20 / \\
8.43-17.57\end{array}$ & 0.49 & 1.329 & $0.779-2.269$ \\
\hline Recessive & $\begin{array}{l}\text { RR vs. } \\
\text { HR/HH }\end{array}$ & $6 / 6$ & $\begin{array}{l}3.52-8.48 / \\
5.16-6.84\end{array}$ & 0.586 & 0.636 & $0.223-1.815$ & $13 / 13$ & $\begin{array}{c}11.76-14.24 / \\
11.66-14.34\end{array}$ & 0.249 & 1.233 & $0.475-3.203$ \\
\hline $\begin{array}{l}\text { Over- } \\
\text { dominant }\end{array}$ & $\begin{array}{l}\text { HR vs. } \\
\mathrm{HH}+\mathrm{RR}\end{array}$ & $7 / 6$ & $\begin{array}{l}5.22-8.78 / \\
4.83-7.17\end{array}$ & 0.824 & 1.162 & $0.687-1.964$ & $13 / 13$ & $\begin{array}{l}10.54-15.46 / \\
12.24-13.77\end{array}$ & 0.866 & 1.239 & $0.726-2.113$ \\
\hline Allele & R vs. H & $6 / 6$ & $\begin{array}{c}4.72-7.28 / \\
4.38-7.62\end{array}$ & 0.86 & 0.989 & $0.733-1.333$ & $13 / 13$ & $\begin{array}{l}11.18-14.82 / \\
10.58-15.42\end{array}$ & 0.357 & 1.191 & $0.870-1.631$ \\
\hline \multicolumn{12}{|c|}{ FCGR3A V158F } \\
\hline \multirow[t]{2}{*}{ Co-dominant } & $\begin{array}{c}\text { FV } \\
\text { vs. FF }\end{array}$ & $6 / 8$ & $\begin{array}{l}4.62-7.39 / \\
4.78-11.23\end{array}$ & 0.619 & 0.845 & $0.453-1.577$ & $14.5 / 14$ & $\begin{array}{c}10.21-18.79 / \\
11.04-16.96\end{array}$ & 0.777 & 1.002 & $0.472-2.127$ \\
\hline & $\begin{array}{c}\text { VV } \\
\text { vs. FF }\end{array}$ & $5.5 / 8$ & $\begin{array}{c}0.00-11.04 / \\
4.78-11.23\end{array}$ & 0.933 & 0.936 & $0.406-2.159$ & $12 / 14$ & $\begin{array}{l}2.50-21.50 / \\
11.04-16.96\end{array}$ & 0.815 & 0.828 & $0.344-1.996$ \\
\hline Dominant & $\begin{array}{l}\text { FV/VV } \\
\text { vs. FF }\end{array}$ & $6 / 8$ & $\begin{array}{l}4.38-7.62 / \\
4.78-11.23\end{array}$ & 0.69 & 0.801 & $0.470-1.365$ & $14.5 / 14$ & $\begin{array}{l}9.39-19.61 / \\
11.04-16.96\end{array}$ & 0.724 & 0.901 & $0.495-1.642$ \\
\hline Recessive & $\begin{array}{l}\text { VV vs. } \\
\text { FF/FV }\end{array}$ & $5.5 / 7$ & $\begin{array}{c}0.00-11.04 / \\
4.54-9.46\end{array}$ & 0.852 & 0.839 & $0.375-1.877$ & $12 / 14$ & $\begin{array}{l}2.50-21.50 / \\
10.98-17.02\end{array}$ & 0.698 & 0.797 & $0.349-1.819$ \\
\hline $\begin{array}{l}\text { Over- } \\
\text { dominant }\end{array}$ & $\begin{array}{l}\text { FV vs. } \\
\text { FF/VV }\end{array}$ & $6 / 8$ & $\begin{array}{l}4.62-7.39 / \\
5.26-10.74\end{array}$ & 0.768 & 0.861 & $0.469-1.581$ & $14.5 / 14$ & $\begin{array}{c}10.21-18.79 / \\
11.78-16.22\end{array}$ & 0.914 & 1.06 & $0.515-2.184$ \\
\hline
\end{tabular}

(Continued) 


\begin{tabular}{|c|c|c|c|c|c|c|c|c|c|c|c|}
\hline \multirow{3}{*}{ Model } & \multirow{3}{*}{ Locus } & \multicolumn{5}{|c|}{ Progression-free survival } & \multicolumn{5}{|c|}{ Overall survival } \\
\hline & & \multicolumn{2}{|c|}{ Months } & \multirow{2}{*}{$\begin{array}{c}P- \\
\text { value }^{*}\end{array}$} & \multicolumn{2}{|r|}{ Cox } & \multicolumn{2}{|c|}{ Months } & \multicolumn{2}{|l|}{$\begin{array}{c}P- \\
\text { value }\end{array}$} & Cox \\
\hline & & Median & $95 \% \mathrm{CI}$ & & $\mathbf{H R}^{\#}$ & $95 \%$ CI & Median & $95 \% \mathrm{CI}$ & & $\mathbf{H R}^{\#}$ & $95 \% \mathrm{CI}$ \\
\hline Allele & V vs. F & $6 / 8$ & $\begin{array}{l}4.18-7.83 / \\
5.68-10.32\end{array}$ & 0.682 & 0.862 & $0.581-1.278$ & $14.5 / 14$ & $\begin{array}{l}8.68-20.32 / \\
11.87-16.13\end{array}$ & 0.632 & 0.888 & $0.585-1.349$ \\
\hline \multicolumn{2}{|c|}{ FCGR combined } & & & & & & & & & & \\
\hline \multicolumn{2}{|c|}{$\mathrm{H}$ and $\mathrm{F}$ vs RR or VV } & $6 / 9$ & $\begin{array}{l}3.92-8.08 / \\
6.87-11.13\end{array}$ & 0.398 & 0.798 & $0.364-1.750$ & $13 / 15$ & $\begin{array}{c}10.21-15.79 / \\
12.51-17.49\end{array}$ & 0.903 & 0.823 & $0.360-1.878$ \\
\hline
\end{tabular}

Abbreviation: $\mathrm{mCRC}$ : metastatic colorectal cancer;

${ }^{*} P$-value: Result of log-rank test of Kaplan-Meier curve;

${ }^{\#}$ HR: hazard ratio adjusted by sex, age, smoking, drinking and status of diabetes and hypertension; CI: confidential interval.
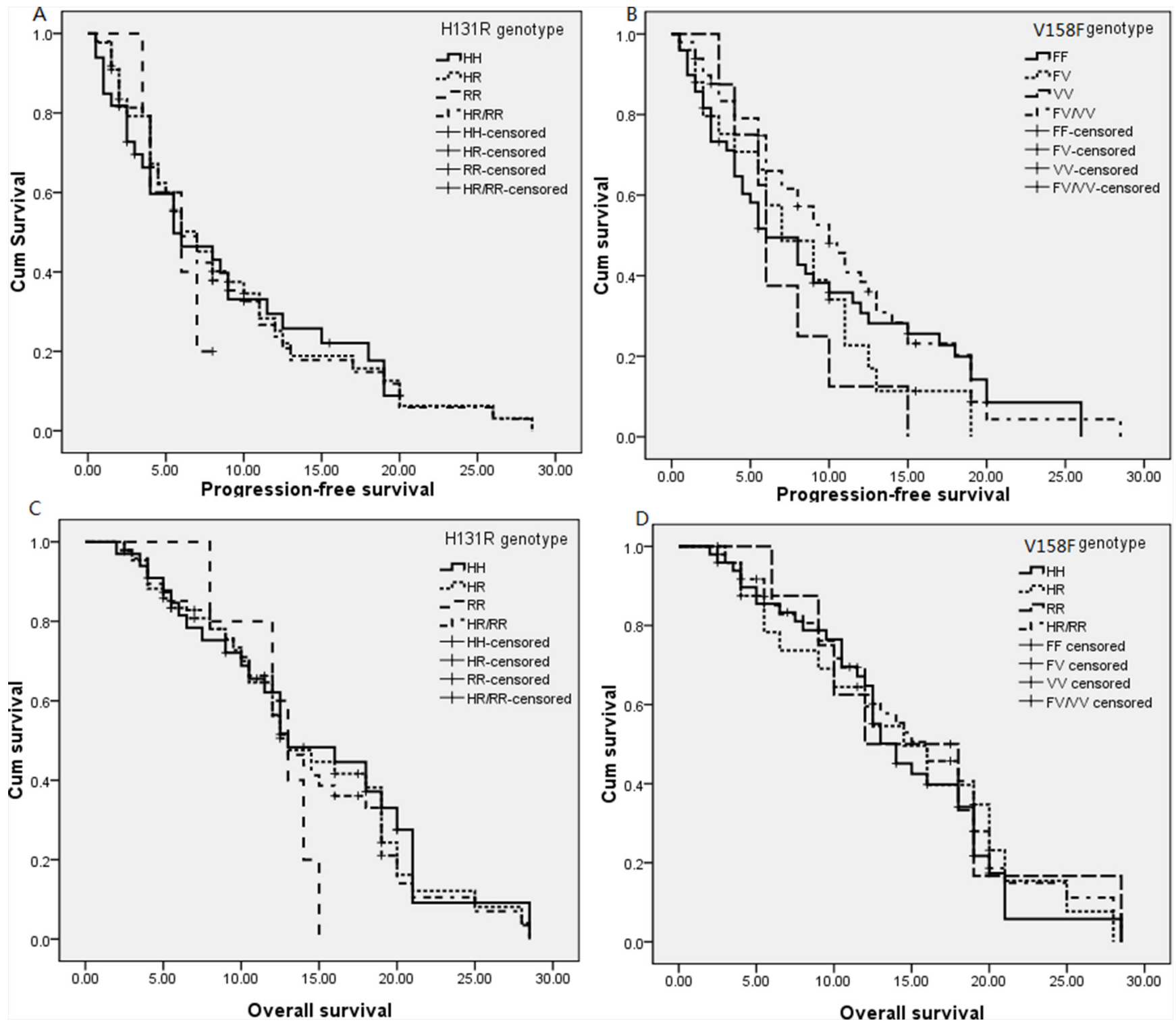

Figure 1: Kaplan-Meier curves for $F C G R 2 A$ H131R and $F C G R 3 A$ V158F for progression-free survival and overall survival. A. H131R for PFS; B. V158F for PFS; C. H131R for OS; D. V158F for OS. 
$\mathrm{RR}(\mathrm{HR}=1.239,95 \% \mathrm{CI}=0.726-2.113)$, allele $\mathrm{R}$ vs. $\mathrm{H}$ $(\mathrm{HR}=1.191,95 \% \mathrm{CI}=0.870-1.631)$, respectively. Patient harbored genotype $\mathrm{FV}(\mathrm{HR}=0.845,95 \% \mathrm{CI}=0.453-1.577$ for $\mathrm{PFS}, \mathrm{HR}=1.002,95 \% \mathrm{CI}=0.472-2.127$ for OS $), \mathrm{VV}(\mathrm{HR}=0.936,95 \% \mathrm{CI}=0.406-2.159$ for PFS, HR $=0.828,95 \% \mathrm{CI}=0.344-1.996$ for $\mathrm{OS})$ and $\mathrm{FV} / \mathrm{VV}(\mathrm{HR}=0.801,95 \% \mathrm{CI}=0.470-1.365$ for $\mathrm{PFS}, \mathrm{HR}=0.901,95 \% \mathrm{CI}=0.495-1.642$ for $\mathrm{OS}$ ) of $\mathrm{V} 158 \mathrm{~F}$ within FCGR3A were not shown a statistically longer or shorter PFS and OS than those individuals harbored genotype FF, respectively, no significant association was observed in recessive $(\mathrm{HR}=0.839,95 \% \mathrm{CI}=0.375-1.877$ for $\mathrm{PFS}$; $\mathrm{HR}=0.797,95 \% \mathrm{CI}=0.349-1.819$ for OS), overdominant $(\mathrm{HR}=0.861,95 \% \mathrm{CI}=0.469-1.581$ for PFS; $\mathrm{HR}=1.06,95 \% \mathrm{CI}=0.515-2.184$ for $\mathrm{OS})$, allele $(\mathrm{HR}=0.862,95 \% \mathrm{CI}=0.581-1.278$ for
PFS; HR $=0.888,95 \% \mathrm{CI}=0.585-1.349$ for OS $)$ models. Meanwhile, PFS $(\mathrm{HR}=0.798,95 \% \mathrm{CI}=0.364-1.750)$ and $\mathrm{OS}(\mathrm{HR}=0.823,95 \% \mathrm{CI}=0.360-1.878)$ of the cases harbored genotype RR or VV weren't shown significant difference when compared to cases with allele $\mathrm{H}$ and $\mathrm{F}$ (Table 3, Figure 1).

A total of 14 published articles (15 eligible studies) and our study were included in this comprehensive metaanalysis to further evaluate the association of $F C G R 2 A$ and FCGR3A polymorphisms with clinical outcome in advanced $\mathrm{CRC}$ patients undergoing anti-EGFR mAb based therapy [18-31]. The baseline characteristics of included studies and the results of heterogeneity test, odds ratio (OR), median survival ratio (MSR) and corresponding 95\% confidential interval $(95 \% \mathrm{CI})$ were reported in Supplementary Table 1 and Table 4 and 5. As shown from Table 4, Genotypes of H131R weren't

Table 4: Meta-analysis results of the association between FCGR2A H131R, FCGR3A F158V polymorphisms and clinical response of $\mathrm{mCRC}$ patients with adjuvant anti-EGFR $\mathrm{mAb}$ therapy

\begin{tabular}{|c|c|c|c|c|c|c|c|c|}
\hline \multirow{2}{*}{ Population } & \multirow{2}{*}{ Model } & \multirow{2}{*}{ Comparison } & \multicolumn{3}{|r|}{ ORR } & \multicolumn{3}{|r|}{ DCR } \\
\hline & & & No. & $P_{\mathrm{h}}$ & OR(95\%CI) & No. & $P_{\mathbf{h}}$ & OR(95\%CI) \\
\hline \multicolumn{9}{|c|}{ FCGR2A H131R } \\
\hline \multirow[t]{6}{*}{ Overall } & Co-dominant & HR vs. HH & 738 & 0.456 & $1.136(0.815-1.584)$ & 297 & 0.987 & $1.244(0.732-2.112)$ \\
\hline & & RR vs. HH & 463 & 0.234 & $1.308(0.852-2.009)$ & 185 & 0.077 & $1.035(0.307-3.495)$ \\
\hline & Dominant & HR/RR vs. HH & 1857 & 0.389 & $1.156(0.919-1.453)$ & 1333 & 0.307 & $0.993(0.780-1.265)$ \\
\hline & Recessive & RR vs.HR/RR & 933 & 0.283 & $1.269(0.908-1.773)$ & 370 & 0.057 & $0.883(0.285-2.732)$ \\
\hline & $\begin{array}{l}\text { Over- } \\
\text { dominant }\end{array}$ & HR vs. HH/RR & 904 & 0.358 & $0.993(0.744-1.325)$ & 370 & 0.79 & $1.242(0.805-1.916)$ \\
\hline & Allele & R vs. H & 1808 & 0.116 & $1.180(0.959-1.452)$ & 740 & 0.002 & $0.780(0.414-1.471)$ \\
\hline \multirow[t]{6}{*}{$K R A S$ wild } & Co-dominant & HR vs. HH & 393 & 0.391 & $1.234(0.831-1.835)$ & 123 & 0.941 & $1.459(0.782-2.722)$ \\
\hline & & RR vs. HH & 246 & 0.184 & $1.234(0.771-1.974)$ & 74 & 0.178 & $1.623(0.646-4.076)$ \\
\hline & Dominant & HR/RR vs. HH & 1110 & 0.316 & $1.247(0.960-1.619)$ & 790 & 0.671 & $1.115(0.819-1.518)$ \\
\hline & Recessive & RR vs.HR/HH & 500 & 0.14 & $1.193(0.752-1.892)$ & 151 & 0.096 & $1.175(0.291-4.748)$ \\
\hline & $\begin{array}{l}\text { Over- } \\
\text { dominant }\end{array}$ & HR vs. HH/RR & 471 & 0.175 & $1.079(0.740-1.571)$ & 151 & 0.422 & $1.323(0.622-2.815)$ \\
\hline & Allele & R vs. $\mathrm{H}$ & 942 & 0.043 & $1.10(0.692-1.748)$ & 302 & 0.084 & $1.304(0.765-2.224)$ \\
\hline \multicolumn{9}{|c|}{ FCGR3A V158F } \\
\hline \multirow[t]{6}{*}{ Overall } & Co-dominant & FV vs. FF & 834 & 0.583 & $0.805(0.592-1.096)$ & 283 & 0.15 & $0.937(0.567-1.550)$ \\
\hline & & VV vs. FF & 561 & 0.644 & $0.881(0.565-1.375)$ & 193 & 0.007 & $1.311(0.292-5.897)$ \\
\hline & Dominant & FV/VV vs. FF & 1026 & 0.113 & $0.880(0.667-1.162)$ & 383 & 0.038 & $0.916(0.576-1.458)$ \\
\hline & Recessive & VV vs. FV/FF & 1983 & 0.874 & $0.987(0.743-1.311)$ & 1331 & 0.041 & $0.897(0.407-1.973)$ \\
\hline & $\begin{array}{l}\text { Over- } \\
\text { dominant }\end{array}$ & FV vs. FF/VV & 977 & 0.735 & $0.855(0.643-1.138)$ & 339 & 0.752 & $0.549-1.390$ \\
\hline & Allele & V vs. F & 1954 & 0.351 & $0.866(0.702-1.068)$ & 678 & 0.013 & $1.019(0.541-1.919)$ \\
\hline
\end{tabular}

(Continued) 


\begin{tabular}{|c|c|c|c|c|c|c|c|c|}
\hline \multirow{2}{*}{ Population } & \multirow{2}{*}{ Model } & \multirow{2}{*}{ Comparison } & \multicolumn{3}{|r|}{ ORR } & \multicolumn{3}{|r|}{ DCR } \\
\hline & & & No. & $P_{\mathrm{h}}$ & OR(95\%CI) & No. & $P_{\mathrm{h}}$ & OR(95\%CI) \\
\hline \multirow[t]{6}{*}{$K R A S$ wild } & Co-dominant & FV vs. FF & 404 & 0.61 & $0.851(0.572-1.266)$ & 119 & 0.052 & $0.898(0.269-2.992)$ \\
\hline & & VV vs. FF & 266 & 0.935 & $1.153(0.640-2.077)$ & 81 & 0.072 & $1.302(0.286-5.940)$ \\
\hline & Dominant & FV/VV vs. FF & 471 & 0.605 & $0.889(0.614-1.287)$ & 154 & 0.024 & $0.987(0.284-3.429)$ \\
\hline & Recessive & VV vs. FV/FF & 1154 & 0.961 & $1.089(0.779-1.522)$ & 815 & 0.283 & $0.710(0.483-1.043)$ \\
\hline & $\begin{array}{l}\text { Over- } \\
\text { dominant }\end{array}$ & FV vs. FF/VV & 471 & 0.528 & $0.887(0.602-1.306)$ & 154 & 0.268 & $0.708(0.332-1.511)$ \\
\hline & Allele & V vs. F & 942 & 0.707 & $0.987(0.744-1.311)$ & 308 & 0.033 & $1.121(0.403-3.119)$ \\
\hline \multicolumn{9}{|c|}{ FCGR combined } \\
\hline \multirow[t]{2}{*}{ Overall } & & $\begin{array}{l}\mathrm{HH} \text { or } \mathrm{VV} \text { vs. } \\
\mathrm{R} / \mathrm{F}\end{array}$ & 160 & 0.126 & $1.698(0.762-3.784)$ & 160 & 0.107 & $1.012(0.524-1.955)$ \\
\hline & & $\begin{array}{l}\text { RR or VV vs. } \\
\mathrm{H} / \mathrm{F}\end{array}$ & 115 & 0.787 & $0.684(0.210-2.222)$ & 115 & 0.001 & $\begin{array}{c}4.103 \\
(0.108-156.599)\end{array}$ \\
\hline
\end{tabular}

Abbreviation: mCRC: metastatic colorectal cancer; $P_{\mathrm{h}}$ : result of heterogeneity test; ORR: objective response rate; DCR: disease control rate; OR: odds ratio; CI: confidential interval;

Table 5: Meta-analysis results of the association of FCGR2A H131R, FCGR3A F158V polymorphisms and clinical survival of $\mathrm{mCRC}$ patients with adjuvant anti-EGFR $\mathrm{mAb}$ therapy

\begin{tabular}{|c|c|c|c|c|c|c|c|c|}
\hline Population & Model & Comparison & PFS & & & OS & & \\
\hline & & & No. & $P_{\mathrm{h}}$ & $\operatorname{MSR}(95 \% \mathrm{CI})$ & No. & $P_{\mathbf{h}}$ & $\operatorname{MSR}(95 \% \mathrm{CI})$ \\
\hline \multicolumn{9}{|c|}{ FCGR2A H131R } \\
\hline \multirow[t]{3}{*}{ Overall } & $\begin{array}{c}\text { Co- } \\
\text { dominant }\end{array}$ & HR vs. HH & 739 & $<0.001$ & $\begin{array}{c}1.111 \\
(0.909-1.358)\end{array}$ & 573 & $<0.001$ & $\begin{array}{c}1.156 \\
(0.929-1.438)\end{array}$ \\
\hline & & RR vs. HH & 466 & $<0.001$ & $\begin{array}{c}0.936 \\
(0.704-1.244)\end{array}$ & 364 & $<0.001$ & $\begin{array}{c}0.864 \\
(0.616-1.212)\end{array}$ \\
\hline & Dominant & HR/RR vs. HH & 1363 & $<0.001$ & $\begin{array}{c}0.993 \\
(0.782-1.262) \\
\end{array}$ & 1176 & 0.822 & $\begin{array}{c}0.946 \\
(0.894-1.002) \\
\end{array}$ \\
\hline \multirow[t]{3}{*}{$K R A S$ wild } & $\begin{array}{c}\text { Co- } \\
\text { dominant }\end{array}$ & HR vs. HH & 471 & $<0.001$ & $\begin{array}{c}1.092 \\
(0.892-1.338)\end{array}$ & 348 & 0.062 & $\begin{array}{c}1.116 \\
(0.942-1.323)\end{array}$ \\
\hline & & RR vs. HH & 285 & 0.015 & $\begin{array}{c}0.953 \\
(0.783-1.159) \\
\end{array}$ & 210 & 0.818 & $\begin{array}{c}0.933 \\
(0.815-1.068) \\
\end{array}$ \\
\hline & Dominant & HR/RR vs. HH & 980 & $<0.001$ & $\begin{array}{c}0.974 \\
(0.778-1.220)\end{array}$ & 821 & 0.044 & $\begin{array}{c}1.056 \\
(0.890-1.253)\end{array}$ \\
\hline \multicolumn{9}{|c|}{$F C G R 3 A$ V158F } \\
\hline \multirow[t]{4}{*}{ Overall } & $\begin{array}{c}\text { Co- } \\
\text { dominant }\end{array}$ & FV vs. FF & 789 & $<0.001$ & $\begin{array}{c}0.943 \\
(0.767-1.159)\end{array}$ & 617 & $<0.001$ & $\begin{array}{c}1.094 \\
(0.907-1.320)\end{array}$ \\
\hline & & VV vs. FF & 514 & $<0.001$ & $\begin{array}{c}0.896 \\
(0.679-1.181)\end{array}$ & 405 & $<0.001$ & $\begin{array}{c}0.733 \\
(0.578-0.930)\end{array}$ \\
\hline & Dominant & FV/VV vs. FF & 311 & 0.027 & $\begin{array}{c}0.680 \\
(0.549-0.842) \\
\end{array}$ & & - & - \\
\hline & Recessive & VV vs. FV/FF & 1077 & $<0.001$ & $\begin{array}{c}1.292 \\
(0.480-3.479)\end{array}$ & & - & - \\
\hline
\end{tabular}

(Continued) 


\begin{tabular}{|c|c|c|c|c|c|c|c|c|}
\hline Population & Model & Comparison & PFS & & & OS & & \\
\hline & & & No. & $P_{\mathrm{h}}$ & MSR(95\%CI) & No. & $P_{\mathrm{h}}$ & $\operatorname{MSR}(95 \% \mathrm{CI})$ \\
\hline \multirow[t]{4}{*}{$K R A S$ wild } & $\begin{array}{c}\text { Co- } \\
\text { dominant }\end{array}$ & FV vs. FF & 478 & $<0.001$ & $\begin{array}{c}1.054 \\
(0.872-1.275)\end{array}$ & 360 & $<0.001$ & $\begin{array}{c}1.128 \\
(0.842-1.510)\end{array}$ \\
\hline & & VV vs. FF & 323 & $<0.001$ & $\begin{array}{c}1.061 \\
(0.733-1.536)\end{array}$ & 244 & $<0.001$ & $\begin{array}{c}0.766 \\
(0.521-1.126) \\
\end{array}$ \\
\hline & Dominant & FV/VV vs. FF & 283 & 0.12 & $\begin{array}{c}0.728 \\
(0.648-0.818)\end{array}$ & & - & - \\
\hline & Recessive & VV vs. FV/FF & 712 & $<0.001$ & $\begin{array}{c}1.382 \\
(0.612-3.12)\end{array}$ & & - & - \\
\hline
\end{tabular}

Abbreviation: mCRC: metastatic colorectal cancer; $P_{\mathrm{h}}$ : result of heterogeneity test; PFS: progression-free survival; OS: overall survival; MSR: median survival ratio; 95\% CI: 95\% confidential interval.

associated with clinical outcome of overall and KRAS wild chemorefractory mCRC patients in terms of ORR, DCR in co-dominant, dominant, recessive, over-dominant, allele models, PFS and OS in co-dominant and dominant models. No significant difference was observed between ORR or DCR and genotypes and alleles of V158F, whatever the KRAS status. However, genotype FV/VV within $\mathrm{V} 158 \mathrm{~F}$ of $F C G R 3 A$ was observed to be significant associated with a shorter PFS in overall (MSR $=0.680$, $95 \% \mathrm{CI}=0.549-0.842)$ and $K R A S$ wild population patients (MSR $=0.728,95 \% \mathrm{CI}=0.648-0.818)$, and individuals harbored genotype FF showed a longer OS than those carrying genotype $\mathrm{VV}$ of $F C G R 3 A \mathrm{~V} 158 \mathrm{~F}$ only in overall population $(\mathrm{MSR}=0.733,95 \% \mathrm{CI}=0.578-0.930)($ Table 5 and Figure 2). There was no significant publication bias in all comparisons between genotypes of H131R and V158F and clinical response and outcome, respectively.

\section{DISCUSSION}

This study, to the best of our knowledge, is the first report that combined a retrospective study and a comprehensive meta-analysis with the largest sample size for estimating the association between FCGR2A H131R and FCGR $3 A \mathrm{~V} 158 \mathrm{~F}$ and clinical outcome of mCRC patients with treatment of anti-EGFR mAb based therapy. With limitation of small sample size, our retrospective study showed no significant association between FCGR2A and $F C G R 3 A$ polymorphisms and clinical outcome in 82 wild-KRAS chemorefractory mCRC individuals treated with chemotherapy plus cetuximab. However, the robust results of meta-analysis showed that genotype FF within V158F was significant associated with a longer PFS and OS than FV/VV and VV genotype in overall and KRAS wild population, respectively. These findings suggested that $K R A S$ wild chemorefractory $\mathrm{mCRC}$ individual harbored genotype FF of V158F could benefit from antiEGFR mAb based therapy in terms of PFS and OS, and the genotype could be used as a genetic biomarker to predict clinical prognosis of wild-KRAS mCRC patients with adjuvant treatment of anti-EGFR $m A b$.

$\mathrm{ADCC}$ is considered as an important mechanism for the antitumor effect of anti-EGFR mAb in mCRC patients $[13,32]$. Cetuximab, an IgG1 monoclonal antibodies, can bind with EGFR protein of tumor cell by its Fab fragment, and its Fc fragment engages FCGR on an immune cell to activate the process of $\mathrm{ADCC}$, eventually resulting in attacking and eliminating the cetuximab-coated tumor cell. Alternation of amino acid at specific binding-domain of Fc fragment of IgG can affected FCGR2A and FCGR3A binding affinity in vitro and in vivo [33-35]. H131A and $\mathrm{V} 158 \mathrm{~F}$ are located at the extracellular ligand-specific binding domain of FCGR $2 A$ and $F C G R 3 A$, respectively. $F C G R 2 A-\mathrm{H}$ allele and $F C G R 3 A-\mathrm{V}$ allele and $\mathrm{VV}$ genotype were associated with higher affinity to $\lg G 2, \lg G 1$ and lgG3 than others [35-37], respectively. Thus, the two common polymorphisms within FCGR2A and FCGR $3 A$ affected their receptor's specific binding-domain of $\mathrm{IgG}$, leading to different antitumor effect in mCRC patients with anti-EGFR mAb based therapy.

Several studies concerning FCGR polymorphisms and efficacy of trantuumab and rituximab demonstrated that FCGR $2 A \mathrm{H} 131 \mathrm{R}$ and FCGR $3 A \mathrm{~V} 158 \mathrm{~F}$ could predict clinical efficacy and survival of patients with breast cancer and lymphoma, respectively $[17,38]$. Cohort study concerned mCRC supported the hypothesis that FCGR $2 A \mathrm{H} 131 \mathrm{R}$ and FCGR $3 A \mathrm{~V} 158 \mathrm{~F}$ were associated with clinical outcome of mCRC patients with cetuximab in a small sample size [30]. Whereas, a large cohort study containing 1123 chemorefractory mCRC patients failed to support the significant results [18]. In our retrospective study, we didn't observe the significant association between FCGR2A H131R and FCGR3A V158F and clinical outcome in chemotherapy plus cetuximab treated mCRC patients in terms of ORR, DCR, PFS and OS in retrospective study. Moreover, H131R within FCGR2A wasn't associated with ORR, DCR, PFS and OS in overall and KRAS wild subgroups in the meta-analysis, 
A

Pander 2010

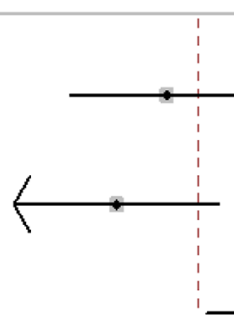

$0.64(0.54,0.77)$

34.56

Park 2012

Ying 2015

Overall (I-squared $=72.4 \%, p=0.027)$

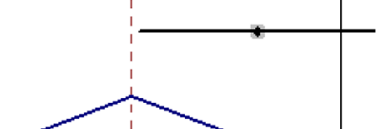

33.80

$0.86(0.69,1.06)$

31.25

$0.68(0.55,0.84)$

100.00

NOTE: Weights are from random effects analysis

\subsection{3}

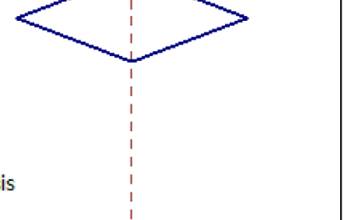

B Study

Pander 2010

Park 2012

Ying 2015

Overall (I-squared $=52.9 \%, p=0.120)$

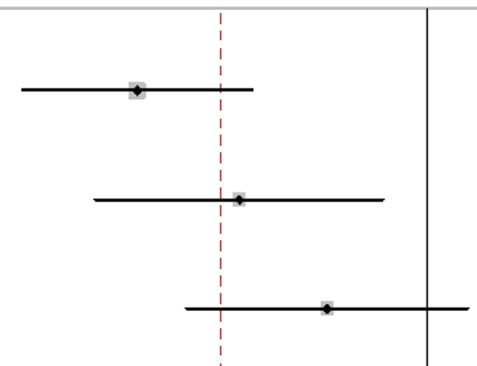

MSR $(95 \%$ CI)

$0.64(0.54,0.77)$

$0.75(0.60,0.93)$

$0.86(0.69,1.06)$

$0.73(0.65,0.82)$

100.00
0.536

C

Study

Zhang 2007

Park 2012

Inoue 2014

Kjersem 2014

Negri 2014

Ying 2015

Overall (I-squred $=79.2 \%, p=0.000$

NOTE: Weights are from random effects analysis

$$
0.361
$$
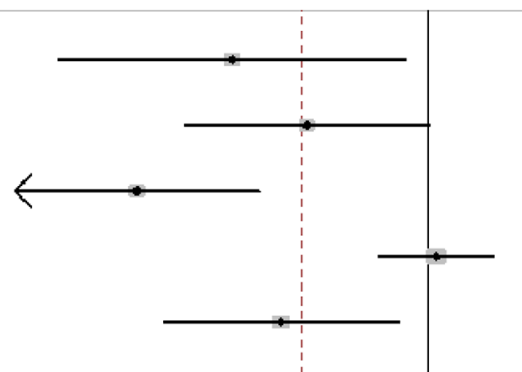

$1.02(0.89,1.17)$

$0.70(0.52,0.93)$

$0.86(0.66,1.11)$

$0.73(0.58,0.93)$

Figure 2: Meta-analysis result of association between $F C G R 3 A$ V158F and progression-free survival and overall survival in mCRC patients treated with anti-EGFR mAb based therapy. A. FV/VV vs. FF for PFS in overall population; B. FV/VV vs. FF for PFS in KRAS wild population; C. VV vs. FF for OS in overall population. 
indicating that the polymorphism of FCGR $2 A$ might be not involved in anti-EGFR $\mathrm{mAb}$ induced ADCC effect and it could not predict clinical outcome of cetuximab based therapy in wild-KRAS mCRC patients. Nonetheless, V158F within FCGR3A was significantly associated with PFS in dominant genetic model in overall and KRAS wild populations and genotype $\mathrm{VV}$ of the loci was significantly associated with a shorter OS in overall population in the meta-analysis. These findings suggested that V158F of FCGR3A was involved in clinical survival of anti-EGFR $\mathrm{mAb}$ treated $\mathrm{mCRC}$ individual, and genotype FF could be used as a prognostic biomarker to predict survival of antiEGFR $\mathrm{mAb}$ treated $\mathrm{mCRC}$ patients in overall population, especially $K R A S$ wild carrier. Our finding is in agreement with previous studies conducted by Zhang et al and Pander et al $[25,30]$, respectively, but against our speculation and inconsistent with the results of other studies [18, 19, 23].

The possible reason why genotype FF of $\mathrm{V} 158 \mathrm{~F}$ within FCGR3A was associated a longer PFS and OS compared with genotype $\mathrm{VV}$ and $\mathrm{VV} / \mathrm{VF}$ remains partly understand. Included individuals in our retrospective study and eligible studies in meta-analysis were all chemorefractory mCRC patients, and long-time's regular chemoradiotherapy and increasing burden of CRC leaded to a gradual decreased immunologic surveillance $[18,26]$. NK cell was generally scarce within CRC tumor tissues, on the contrary, normal level of NK cell was examined in adjacent normal mucosa [39]. Meanwhile, a significant reduction in the percentage of $\mathrm{NKG} 2 \mathrm{D}+\mathrm{NK}$ cell was observed in peripheral blood of metastatic colon cancer patients [40]. Additionally, tumor-associated macrophage (TAM) is one of the most frequently found immune cells in the tumor microenvironment [41]. Although genotype VV of V158F displayed a significant higher anti-EGFR $\mathrm{mAb}$-triggered ADCC in peripheral blood mononuclear cells in vitro [23]. However, a possible mechanism is that the high-affinity allele $\mathrm{V}$ of $\mathrm{V} 158 \mathrm{~F}$ within $F C G R 3 A$ might contribute to decreased ADCC-triggered by NK cell [18, 26], but through cross-linking of the FCGR to increase activation of TAM in tumor microenvironment by antiEGFR mAb. Activated TAM releases large amounts of pro-angiogentic and pro-metastatic regulators to facilitate angiopoiesis, matrix breakdown, tumor cell motility and promote tumor growth, leading to poor prognosis $[41,42]$.

In conclusion, we fail to find the significant association between FCGR2A H131R and clinical outcome in $K R A S$ wild $\mathrm{mCRC}$ individuals with adjuvant cetuximab therapy, but $K R A S$ wild chemorefractory $\mathrm{mCRC}$ individual harbored genotype FF of V158F can benefit from cetuximab based therapy in terms of PFS and OS, and it may be a prognostic factor to evaluate the clinical survival of wild-KRAS mCRC patients undergoing anti-EGFR mAb therapy. With limitation of the study, multi-central, well designed prospective studies with large sample size are warrant to further validate our findings.

\section{MATERIALS AND METHODS}

\section{Patients}

Patients with mCRC who diagnosed and treated in Nanjing First Hospital and Pingxiang People's Hospital between 2007 May and 2014 December were enrolled in our retrospective study. All of them were histologically proven TNM-IV stage and harbored with wild-KRAS. The included individuals were treated with cetuximab plus conventional chemotherapy regimen such as CapeOX, FOLFIR1 and FOLFOX. Cetuximab was used $400 \mathrm{mg} / \mathrm{m}^{2}$ dose at first, then $250 \mathrm{mg} / \mathrm{m}^{2}$ subsequently, 21 day a cycle, and treated until cancer progression or unacceptable toxicity. This study was approved by the Institution Ethics Commission of Southeast University, and all included participants were signed informed consents.

\section{Efficacy evaluation and following-up}

In accordance with the Response Evaluation Criteria In Solid Tumors Criteria 1.0 (RECST 1.0), the cancer response to cetuximab therapy was evaluated every month at the time of hospitalization and the evaluated results were defined as complete response (CR), partial response $(\mathrm{PR})$, stable disease (SD) and progressive disease (PD), respectively. Progression-free survival (PFS), overall survival (OS) and objective response rate (ORR) and disease control rate (DCR) were used as endpoints in our study. PFS and OS were calculated from the first date of cetuximab usage to the time of disease progression and death, respectively. Relevant data (sex, age, treatment, $K R A S$ status, and response evaluated results) were collected from the cases' medical records.

\section{PCR amplification and genotyping}

Human genomic DNA was extracted from paraffinembedded CRC tissues or EDTA anti-coagulated peripheral blood samples using TIANamp Genomic DNA Kit (TIANGEN, Beijing, China) and stored at $-80^{\circ} \mathrm{C}$ till detection. Taqman-genotyping real-time PCR were selected to detect genotypes of FCGR2A H131R and FCGR3A V158F using ABI7500 fluorescence quantitative PCR system (Applied Biosystems, Foster City, USA). The detail probe, primer sequence, PCR protocol and detection were in accordance to the study reported by Norton et al [38].

\section{Meta-analysis}

In order to enhance statistical power of the study, a meta-analysis including all eligible studies was conducted to further confirm our results. Relevant article was screened in the Pubmed, Web of SCI and Wanfang databases in English and Chinese using search terms of 
"FCGR $2 A / 3 A$ and cetuximab or CRC", "rs1801274/ rs396991 and cetuximab or CRC", and "H131R/V158F and cetuximab or CRC" dating end up to March of 2015. In addition, a manual searching in reference of relevant articles was carried out to obtain substantial studies. Relevant articles were identified as eligible study in accordance with following including criteria: (1) retrospective or prospective study was concerned FCGR $2 A / 3 A$ polymorphisms and clinical outcome of chemorefractory $\mathrm{mCRC}$ cases treated with adjuvant cetuximab"; (2) efficacy evaluation criteria was in accordance with RECST 1.0/1.1; (3) studies provided median survival time, number of cases, genotype distributions, ORR, DCR. On the contrary, review, correspondence, letter, meta-analysis, case report or studies without providing relevant data were excluded from the study. Relevant study search, eligible study identification, data extraction and statistics were conducted by two independent investigator (Hou-Qun Ying and Feng Wang) and any discrepancies were discussed to reach consensus or made final decision by the third investigator (Xiao-Lin Chen).

\section{Statistical analysis}

The genotype frequencies of FCGR $2 A$ H131R and $F C G R 3 A$ V158F in all cases were calculated by counting. Pearson $\chi^{2}$ test was selected to evaluate association between the loci genotypes and response to cetuximab based treatment, and $P<0.05$ were considered statistically significance. Kaplan-Meier curve with log-rank test and backward elimination multivariate Cox regression analysis were used to determine the influence of the FCGR2A and FCGR3A polymorphisms on PFS and OS, and the significant $P$-value was set at 0.05 . Q test and estimated $\mathrm{I}^{2}$ were used to assess the heterogeneity between eligible studies, and $P_{\mathrm{h}}<0.1$ or $\mathrm{I}^{2}>50 \%$ was considered as exist of significant heterogeneity. Overall effect of the metaanalysis was evaluated using $\mathrm{Z}$ test in the fixed model $\left(P_{\mathrm{h}}>0.1\right)$ or the random model $\left(P_{\mathrm{h}}<0.1\right)$ according to the heterogeneity test and $P_{\mathrm{z}}<0.05$ showed a statistical significance. Begg's funnel plot was selected to estimate the possible publication bias, and asymmetric funnel plot was considered as the existence of publication bias. All statistical analyses were performed using the SPSS statistical 17.0 (SPSS Inc., Chicago, IL) and Stata 11.0 software (Stata Corporation, College Station, TX).

\section{ACKNOWLEDGMENTS}

This study was supported by the Fundamental Research Funds for the Central Universities, University Graduate Student Scientific Innovation Project of Jiangsu (No.KYLX_0201), National Natural Science Foundation of China (No. 81172141), Nanjing Health Young Talent Project, Nanjing Medical Science and Technique
Development Foundation to Y.Q.P. (No. QRX11255) and B.S.H. (No. QRX11254).

\section{CONFLICTS OF INTEREST}

The authors have declared no conflict of interests with respect to the authorship and/or publication of this article.

\section{REFERENCES}

1. Chen W, Zheng R, Zeng H, Zhang S, He J. Annual report on status of cancer in China, 2011. Chin J Cancer Res. 2015; 27:2-12.

2. Siegel R, Desantis C, Jemal A. Colorectal cancer statistics, 2014. CA Cancer J Clin. 2014; 64:104-117.

3. Goffin JR, Zbuk K. Epidermal growth factor receptor: pathway, therapies, and pipeline. Clin Ther. 2013; 35:1282-1303.

4. Benson AB 3rd, Venook AP, Bekaii-Saab T, Chan E, Chen YJ, Cooper HS, Engstrom PF, Enzinger PC, Fenton MJ, Fuchs CS, Grem JL, Hunt S, Kamel A, Leong LA, Lin E, Messersmith W, et al. Colon cancer, version 3. 2014. J Natl Compr Canc Netw. 2014; 12:1028-1059.

5. Cheng L, Ren W, Xie L, Li M, Liu J, Hu J, Liu BR, Qian XP. Anti-EGFR MoAb treatment in colorectal cancer: limitations, controversies, and contradictories. Cancer Chemother Pharmacol. 2014; 74:1-13.

6. Lyseng-Williamson KA. Cetuximab: a guide to its use in combination with FOLFIRI in the first-line treatment of metastatic colorectal cancer in the USA. Mol Diagn Ther. 2012; 16:317-322.

7. Ciombor KK, Bekaii-Saab T. Emerging treatments in recurrent and metastatic colorectal cancer. J Natl Compr Canc Netw. 2013; 11:Suppl 4:S18-27.

8. Bardelli A, Siena S. Molecular mechanisms of resistance to cetuximab and panitumumab in colorectal cancer. J Clin Oncol. 2010; 28:1254-1261.

9. Ng KP, Hillmer AM, Chuah CT, Juan WC, Ko TK, Teo AS, Ariyaratne PN, Takahashi N, Sawada K, Fei Y, Soh S, Lee WH, Huang JW, Allen JC, Woo XY, Nagarajan N, et al. A common BIM deletion polymorphism mediates intrinsic resistance and inferior responses to tyrosine kinase inhibitors in cancer. Nat Med. 2012; 18:521-528.

10. Vincenzi B, Zoccoli A, Pantano F, Venditti O, Galluzzo S. Cetuximab: from bench to bedside. Curr Cancer Drug Targets. 2010; 10:80-95.

11. Sartore-Bianchi A, Martini M, Molinari F, Veronese S, Nichelatti M, Artale S, Di Nicolantonio F, Saletti P, De Dosso S, Mazzucchelli L, Frattini M, Siena S, Bardelli A. PIK3CA mutations in colorectal cancer are associated with clinical resistance to EGFR-targeted monoclonal antibodies. Cancer Res. 2009; 69:1851-1857. 
12. Qiu LX, Mao C, Zhang J, Zhu XD, Liao RY, Xue K, Li J, Chen Q. Predictive and prognostic value of KRAS mutations in metastatic colorectal cancer patients treated with cetuximab: a meta-analysis of 22 studies. Eur J Cancer. 2010; 46:2781-2787.

13. De Roock W, Jonker DJ, Di Nicolantonio F, SartoreBianchi A, Tu D, Siena S, Lamba S, Arena S, Frattini M, Piessevaux H, Van Cutsem E, O'Callaghan CJ, KhambataFord S, Zalcberg JR, Simes J, Karapetis CS, et al. Association of KRAS p.G13D mutation with outcome in patients with chemotherapy-refractory metastatic colorectal cancer treated with cetuximab. JAMA. 2010; 304:1812-1820.

14. Taylor RP, Lindorfer MA. Analyses of CD20 monoclonal antibody-mediated tumor cell killing mechanisms: rational design of dosing strategies. Mol Pharmacol. 2014; 86:485-491.

15. Kim JW, Kim JH, Im SA, Kim YJ, Han HS, Kim JS, Han SW, Jeon YK, Oh DY, Han W, Kim TY, Park IA, Noh DY, Bang YJ. ABCB1, FCGR2A, and FCGR3A polymorphisms in patients with HER2-positive metastatic breast cancer who were treated with first-line taxane plus trastuzumab chemotherapy. Oncology. 2012; 83:218-227.

16. Persky DO, Dornan D, Goldman BH, Braziel RM, Fisher RI, Leblanc M, Maloney DG, Press OW, Miller TP, Rimsza LM. Fc gamma receptor 3a genotype predicts overall survival in follicular lymphoma patients treated on SWOG trials with combined monoclonal antibody plus chemotherapy but not chemotherapy alone. Haematologica. 2012; 97:937-942.

17. Roca L, Dieras V, Roche H, Lappartient E, Kerbrat P, Cany L, Chieze S, Canon JL, Spielmann M, PenaultLlorca F, Martin AL, Mesleard C, Lemonnier J, de Cremoux P. Correlation of HER2, FCGR2A, and FCGR3A gene polymorphisms with trastuzumab related cardiac toxicity and efficacy in a subgroup of patients from UNICANCER-PACS 04 trial. Breast Cancer Res Treat. 2013; 139:789-800.

18. Geva R, Vecchione L, Kalogeras KT, Vittrup Jensen B, Lenz HJ, Yoshino T, Paez D, Montagut C, Souglakos J, Cappuzzo F, Cervantes A, Frattini M, Fountzilas G, Johansen JS, Hogdall EV, Zhang W, et al. FCGR polymorphisms and cetuximab efficacy in chemorefractory metastatic colorectal cancer: an international consortium study. Gut. 2014.

19. Kjersem JB, Skovlund E, Ikdahl T, Guren T, Kersten C, Dalsgaard AM, Yilmaz MK, Fokstuen T, Tveit KM, Kure EH. FCGR2A and FCGR3A polymorphisms and clinical outcome in metastatic colorectal cancer patients treated with first-line 5-fluorouracil/folinic acid and oxaliplatin +/- cetuximab. BMC Cancer. 2014; 14:340.

20. Inoue $\mathrm{Y}$, Hazama S, Iwamoto S, Miyake $\mathrm{Y}$, Matsuda $\mathrm{C}$, Tsunedomi R, Okayama N, Hinoda Y, Yamasaki T, Suehiro Y, Yoshino S, Sakamoto J, Mishima H, Oka M.
FcgammaR and EGFR polymorphisms as predictive markers of cetuximab efficacy in metastatic colorectal cancer. Mol Diagn Ther. 2014; 18:541-548.

21. Dahan L, Norguet E, Etienne-Grimaldi MC, Formento JL, Gasmi M, Nanni I, Gaudart J, Garcia S, Ouafik L, Seitz JF, Milano G. Pharmacogenetic profiling and cetuximab outcome in patients with advanced colorectal cancer. BMC Cancer. 2011; 11:496.

22. Etienne-Grimaldi MC, Bennouna J, Formento JL, Douillard JY, Francoual M, Hennebelle I, Chatelut E, Francois E, Faroux R, El Hannani C, Jacob JH, Milano G. Multifactorial pharmacogenetic analysis in colorectal cancer patients receiving 5-fluorouracil-based therapy together with cetuximab-irinotecan. Br J Clin Pharmacol. 2012; 73:776-785.

23. Negri FV, Musolino A, Naldi N, Bortesi B, Missale G, Laccabue D, Zerbini A, Camisa R, Chernyschova N, Bisagni G, Loupakis F, Ruzzo A, Neri TM, Ardizzoni A. Role of immunoglobulin $\mathrm{G}$ fragment $\mathrm{C}$ receptor polymorphism-mediated antibody-dependant cellular cytotoxicity in colorectal cancer treated with cetuximab therapy. Pharmacogenomics J. 2014; 14:14-19.

24. Paez D, Pare L, Espinosa I, Salazar J, del Rio E, Barnadas A, Marcuello E, Baiget M. Immunoglobulin G fragment $\mathrm{C}$ receptor polymorphisms and KRAS mutations: are they useful biomarkers of clinical outcome in advanced colorectal cancer treated with anti-EGFR-based therapy?. Cancer Sci. 2010; 101:2048-2053.

25. Pander J, Gelderblom H, Antonini NF, Tol J, van Krieken JH, van der Straaten T, Punt CJ, Guchelaar HJ. Correlation of FCGR3A and EGFR germline polymorphisms with the efficacy of cetuximab in KRAS wild-type metastatic colorectal cancer. Eur J Cancer. 2010; 46:1829-1834.

26. Park SJ, Hong YS, Lee JL, Ryu MH, Chang HM, Kim KP, Ahn YC, Na YS, Jin DH, Yu CS, Kim JC, Kang YK, Kim TW. Genetic polymorphisms of FcgammaRIIa and FcgammaRIIIa are not predictive of clinical outcomes after cetuximab plus irinotecan chemotherapy in patients with metastatic colorectal cancer. Oncology. 2012; 82:83-89.

27. Rodriguez J, Zarate R, Bandres E, Boni V, Hernandez A, Sola JJ, Honorato B, Bitarte J, Garcia-Foncillas N. Fc gamma receptor polymorphisms as predictive markers of Cetuximab efficacy in epidermal growth factor receptor downstream-mutated metastatic colorectal cancer. Eur J Cancer. 2012; 48:1774-1780.

28. Saito R, Suzuki H, Yamada T, Endo S, Moriwaki T, Ueno T, Hirose M, Hirai S, Yamato K, Mizokami Y, Hyodo I. Predicting skin toxicity according to EGFR polymorphisms in patients with colorectal cancer receiving antibody against EGFR. Anticancer Res. 2013; 33:4995-4998.

29. Zhang W, Azuma M, Lurje G, Gordon MA, Yang D, Pohl A, Ning Y, Bohanes P, Gerger A, Winder T, Hollywood E, Danenberg KD, Saltz L, Lenz HJ. Molecular 
predictors of combination targeted therapies (cetuximab, bevacizumab) in irinotecan-refractory colorectal cancer (BOND-2 study). Anticancer Res. 2010; 30:4209-4217.

30. Zhang W, Gordon M, Schultheis AM, Yang DY, Nagashima F, Azuma M, Chang HM, Borucka E, Lurje G, Sherrod AE, Iqbal S, Groshen S, Lenz HJ. FCGR2A and FCGR3A polymorphisms associated with clinical outcome of epidermal growth factor receptor expressing metastatic colorectal cancer patients treated with single-agent cetuximab. J Clin Oncol. 2007; 25:3712-3718.

31. Bibeau F, Lopez-Crapez E, Di Fiore F, Thezenas S, Ychou M, Blanchard F, Lamy A, Penault-Llorca F, Frebourg T, Michel P, Sabourin JC, Boissiere-Michot F. Impact of Fc \{gamma\}RIIa-Fc \{gamma\}RIIIa polymorphisms and KRAS mutations on the clinical outcome of patients with metastatic colorectal cancer treated with cetuximab plus irinotecan. J Clin Oncol. 2009; 27:1122-1129.

32. Monteverde M, Milano G, Strola G, Maffi M, Lattanzio L, Vivenza D, Tonissi F, Merlano M, Lo Nigro C. The relevance of ADCC for EGFR targeting: A review of the literature and a clinically-applicable method of assessment in patients. Crit Rev Oncol Hematol. 2015.

33. Koene HR, Kleijer M, Algra J, Roos D, von dem Borne AE, de Haas M. Fc gammaRIIIa-158V/F polymorphism influences the binding of $\operatorname{IgG}$ by natural killer cell $\mathrm{Fc}$ gammaRIIIa, independently of the Fc gammaRIIIa48L/R/H phenotype. Blood. 1997; 90:1109-1114.

34. de Haas M, Koene HR, Kleijer M, de Vries E, Simsek S, van Tol MJ, Roos D, von dem Borne AE. A triallelic Fc gamma receptor type IIIA polymorphism influences the binding of human IgG by NK cell Fc gamma RIIIa. J Immunol. 1996; 156:2948-2955.

35. Wu J, Edberg JC, Redecha PB, Bansal V, Guyre PM, Coleman K, Salmon JE, Kimberly RP. A novel polymorphism of FcgammaRIIIa (CD16) alters receptor function and predisposes to autoimmune disease. J Clin Invest. 1997; 100:1059-1070.

36. Warmerdam PA, van de Winkel JG, Vlug A, Westerdaal NA, Capel PJ. A single amino acid in the second Ig-like domain of the human Fc gamma receptor II is critical for human IgG2 binding. J Immunol. 1991; 147:1338-1343.

37. Parren PW, Warmerdam PA, Boeije LC, Arts J, Westerdaal NA, Vlug A, Capel PJ, Aarden LA, van de Winkel JG. On the interaction of IgG subclasses with the low affinity Fc gamma RIIa (CD32) on human monocytes, neutrophils, and platelets. Analysis of a functional polymorphism to human IgG2. J Clin Invest. 1992; 90:1537-1546.

38. Norton N, Olson RM, Pegram M, Tenner K, Ballman KV, Clynes R, Knutson KL, Perez EA. Association studies of Fcgamma receptor polymorphisms with outcome in HER2+ breast cancer patients treated with trastuzumab in NCCTG (Alliance) Trial N9831. Cancer Immunol Res. 2014; 2:962-969.

39. Halama N, Braun M, Kahlert C, Spille A, Quack C, Rahbari N, Koch M, Weitz J, Kloor M, Zoernig I, Schirmacher P, Brand K, Grabe N, Falk CS. Natural killer cells are scarce in colorectal carcinoma tissue despite high levels of chemokines and cytokines. Clin Cancer Res. 2011; 17:678-689.

40. Gharagozloo M, Kalantari H, Rezaei A, Maracy MR, Salehi M, Bahador A, Hassannejad N, Narimani M, Sanei MH, Bayat B, Ghazanfari H. The decrease in NKG2D+ Natural Killer cells in peripheral blood of patients with metastatic colorectal cancer. Bratisl Lek Listy. 2015; 116:296-301.

41. Grivennikov SI, Greten FR, Karin M. Immunity, inflammation, and cancer. Cell. 2010; 140:883-899.

42. Pollard JW. Tumour-educated macrophages promote tumour progression and metastasis. Nat Rev Cancer. 2004; 4:71-78. 